\title{
Sequential limit analysis of pipe-soil interaction during large-amplitude cyclic lateral displacements
}

\author{
Deqiong Kong \\ Department of Civil Engineering, City University London, EC1V OHB, UK \\ Chris M. Martin \\ Department of Engineering Science, University of Oxford, Parks Road, Oxford, OX1 3PJ, UK \\ Byron W. Byrne \\ Department of Engineering Science, University of Oxford, Parks Road, Oxford, OX1 3PJ, UK
} 3

ABSTRACT

A newly developed sequential limit analysis (SLA) technique is used to perform largedisplacement numerical simulations relevant to thermally-induced lateral buckling of untrenched subsea pipelines. A rigid plane-strain pipe segment, partially embedded in undrained clay, is subjected to cyclic lateral displacements with amplitudes of up to eight pipe diameters. A constant vertical dead load is applied to the pipe during each lateral sweep, but in some analyses this load is varied from one sweep to the next. The SLA method directly models the evolution of the soil surface profile, including active and dormant berms, and incorporates strain softening behaviour caused by soil remoulding. Comparisons of the numerical results with published centrifuge model test data, for a range of loading cases, are provided in terms of the pipe invert trajectory and lateral soil resistance. There is good overall agreement between the numerical and experimental results, demonstrating the suitability of SLA for solving such problems. Detailed aspects of the cyclic loading behaviour are discussed with reference to soil failure mechanisms and bearing capacity failure envelopes for combined vertical and horizontal loading. Finally, two brief parametric studies are used to explore the effects of the initial pipe embedment and vertical loading history on the subsequent lateral loading behaviour. 
1

2 Offshore oil and gas pipelines are frequently required to operate at high pressure and high 3

\section{INTRODUCTION} temperature $(\mathrm{HPHT})$ relative to the ambient conditions at the seabed. Because of frictional restraint from the adjacent soil, thermally-induced axial expansion of an HPHT pipeline causes axial compressive forces to build up along the pipe. These forces commonly reach a magnitude where the pipeline (a slender structural element) becomes vulnerable to buckling. Economic and practical considerations mean that in deep water, HPHT pipelines are generally laid directly on the seabed rather than being trenched. For these on-bottom pipelines, thermally-induced buckling tends to occur in the horizontal (lateral) direction across the seabed surface.

A buckling event can involve lateral displacements of up to 10 or 20 pipe diameters (Bruton et al., 2007). As a result of periodic start-ups and shut-downs, and there can be 100 to 1000 cycles of motion during the 20 year life span of a typical HPHT pipeline (Cheuk et al., 2007). The buckling displacement gives rise to significant bending moments at the crown of the buckle, sometimes sufficient to cause plastic strain within the pipe wall, and introducing risks of a pipeline failure (Bruton et al., 2007). Despite the fact that various guidelines have been proposed for the design of HPHT pipelines, there are at least three known failures, in the North Sea and off West Africa and Brazil, caused by lateral buckling (Bruton et al., 2005). Since any failure of the pipeline can result in containment loss, and a severe environmental hazard (Almeida et al., 2001), it is essential that pipeline designers have a clear understanding of, and are able to model accurately, the pipe-soil interaction that occurs during lateral buckling.

An elegant solution to this problem is to work with the pipeline rather than against it, by encouraging the formation of lateral buckles at predetermined points along the length of the pipeline (Bruton et al., 2005). This is known as controlled lateral buckling, and requires a comprehensive understanding of pipe-soil interaction, especially in the lateral direction (Bruton et al., 2005, 2007). A key aspect is the prediction of the soil resistance encountered by the laterally moving pipe. However this is an area of great uncertainty, especially in soft clay soils, which are dominant across large regions of deep-water offshore development. Given the nature 
of pipeline buckling, it is not possible to adopt a conservative assessment of soil response because both upper and lower estimates of the lateral soil resistance are important. If the resistance cannot be quantified accurately, then a controlled lateral buckling solution cannot be developed with confidence, and there may be operational problems such as planned buckles failing to initiate, or 'rogue' buckles forming at unplanned locations (Urthaler et al., 2012).

Theoretical approaches to pipe-soil interaction on undrained clay have been widely pursued (Randolph \& Houlsby, 1984; Murff et al., 1989; Aubeny et al., 2005; Martin \& Randolph, 2006; Randolph \& White, 2008a, b) and these form the basis for much of the on-bottom stability analysis of pipelines. However, the substantial changes in seabed geometry and soil strength, as the pipeline scrapes across the surface and ploughs the soil into a 'berm' that is pushed ahead of the pipe, cannot reasonably be accounted for in these models. Recent physical model tests of pipe segments on clay have provided more insight into soil failure mechanisms, as well as the soil resistance mobilised during large lateral pipe movements, providing a basis for empirical force-displacement relationships (Cheuk et al., 2007; Dingle et al., 2008; Cheuk \& White, 2009; White \& Dingle, 2011; Rismanchian, 2014). Inevitably however, these results are limited to specific soil strength profiles and cannot readily be generalised. Numerical analyses have also been widely applied to this problem, with a number of finite element approaches being employed: arbitrary Lagrangian-Eulerian (ALE) (Hesar, 2004; Konuk \& Yu, 2007), coupled Eulerian-Lagrangian (CEL) (Martin et al., 2013; Dutta et al., 2015) and the remeshing and interpolation technique with small strain (RITSS) (Hu \& Randolph, 1998; Wang et al., 2009; Chatterjee et al., 2012; Chatterjee, 2012).

Most of the numerical work reviewed in the previous paragraph has focused on monotonic lateral loading of a pipe segment at constant vertical displacement or under constant vertical load, so that detailed understanding of the cyclic loading response remains limited. In particular, during a cyclic lateral displacement sequence, realistic numerical simulation of the interaction between the 'active' berm (currently being pushed forward by the pipe) and a 'dormant' berm (one left behind by previous movements) poses great challenges. Recently, the behaviour of a pipeline subject to several cycles of lateral motion has been studied by Konuk \& Yu (2007) and 
Sabetamal (2014) using ALE finite element analysis, and by Macaro (2015) using the discrete element method. However these types of analyses have a high computational cost and have therefore been limited to fairly small lateral displacement amplitudes and small numbers of cycles, not fully capturing the potential range of motions experienced by an HPHT offshore pipeline undergoing (cyclic) lateral buckling.

(1)

\section{METHODOLOGY}

\section{Sequential limit analysis}

The numerical study reported this paper was performed using a recently developed sequential limit analysis (SLA) method. The main features of the method are briefly outlined below. More detailed descriptions, as well as a range of verification and validation examples, can be found in Kong (2015) and Kong et al. (2016). Essentially, the SLA approach solves a large-displacement plastic deformation problem by breaking it down into a series of consecutive small-displacement limit analysis problems, with the geometry of the deforming domain (and possibly the material properties) being updated at each increment. This approach had previously been adopted for relatively simple structural problems such as frames and plates (Yang, 1993; Hwan, 1997; Raithatha \& Duncan, 2008) before it was further developed by Kong (2015) to perform twodimensional (plane strain) analyses involving large plastic deformations of a purely cohesive continuum interacting with rigid bodies such as penetrometers and shallow foundations.

The process of SLA modelling involves calculating, using an upper bound limit analysis solution, the velocity field for regions of plastic deformation. This velocity field is then integrated over a small time increment to yield the instantaneous displacement field, which in turn is used to update the model configuration. The process is then repeated for the next increment of the analysis, allowing arbitrarily large plastic deformations to be modelled. The effects of strain rate and strain softening on soil strength can be accommodated through updates to the local material strength throughout the modelled domain (Einav \& Randolph, 2005). 
1 Figure 1 shows a flowchart for the SLA process used in this paper. The in-house finite element

2 limit analysis code OxLim is employed (Makrodimopoulos \& Martin, 2006, 2007, 2008; Martin,

3 2011), along with Triangle (Shewchuk, 2002) for unstructured triangular mesh generation.

4 During an SLA simulation, remeshing is conducted as required to preserve solution accuracy

5 and avoid excessive element distortion. The overall process is controlled using a Python script

6 (Kong, 2015). For a given finite element discretisation, OxLim implements the upper-bound

7 theorem of plasticity to generate a standard second-order cone programming problem, which is

8 solved using the optimisation software MOSEK (MOSEK ApS, 2010). The SLA method has

9 similarities to the RITSS method (Hu \& Randolph, 1998; Wang et al., 2009; Chatterjee et al., 2012) except that at each increment the RITSS method requires the solution of a conventional elastoplastic finite element problem, rather than a numerical optimisation problem.

\section{General features of simulations}

A typical initial configuration of the SLA model, showing the dimensions of the soil domain and the initial mesh, is illustrated in Fig. 2. The resultant forces on the pipe $(V, H)$ and the corresponding displacements $(w, u)$ are also indicated. An adaptive remeshing strategy was used to improve computational efficiency and maintain accuracy throughout the analyses. The soil regions with limited shearing were discretised using a coarse mesh, while regions with intense shearing were discretised using a finer mesh. This was achieved by setting the target element area of a region to be $f A_{\min }$, where $A_{\min }$ is the minimum target element area and $f(\geq 1)$ is a function of the shear strain rate $\dot{\gamma}$ (obtained from the previous increment) and the accumulated plastic shear strain $\xi$. The function is defined so that regions with high values of $\dot{\gamma}$ or $\xi$ are assigned small target element areas, and are therefore discretised with a fine mesh. More details are provided in Kong (2015) and Kong et al. (2016). During each analysis the fine mesh 'followed' the zone(s) of intense shearing as the pipe moved, so that the simulation was conducted without using the fine mesh everywhere. This feature is demonstrated in the examples presented later. The dimensions given in Fig. 2 were varied depending on the example, but were always sufficient to contain all plastic shearing throughout the simulation. 
1 Fixed boundary conditions were applied to the base and sides of the soil domain, whilst the top 2 was set as a free surface. The plane-strain pipe section was modelled as a rigid body of 3 diameter $D$, with the circle approximated by a polygon of 60 straight-line segments. The pipe 4 was free to move in the vertical and lateral directions only (i.e. rotation was prevented). The 5 incremental pipe displacement, $\delta d$, that was used to update each analysis increment was set as $60.0025 D$ for vertical loading and $0.005 D$ for lateral loading. The minimum target element area,

$7 A_{\min }$, for the adaptive mesh generation was set as $0.0005 D^{2}$. These values of $\delta d$ and $A_{\min }$ were determined by Kong (2015) to produce robust results for this type of SLA simulation.

The soil was modelled as a rigid-plastic Tresca material, with an intact shear strength defined in the initial model configuration as $s_{u}=s_{u m}+\rho z$. Here $s_{u m}$ is the undrained shear strength at the mudline (initial undeformed soil surface), $\rho$ is the strength gradient and $z$ is the depth below the mudline. A no-tension condition was imposed at the pipe-soil interface to allow separation to occur, for example when lateral pipe movement takes place. This separation or 'breakout' behaviour has been observed in numerous physical modelling studies (Bruton et al., 2005; Cheuk, 2005; Dingle et al., 2008; White \& Dingle, 2011; Rismanchian, 2014). The shear stress mobilised at the interface was limited to $\tau_{\text {int }}=\alpha s_{u}$, where $\alpha$ is the roughness coefficient of the interface and $s_{u}$ is the operative shear strength of the adjacent soil. To account for the effect of strain softening on the soil strength, a simplified form of the total stress constitutive model proposed by Einav \& Randolph (2005) was employed:

$$
s_{\mathrm{u}}=s_{\mathrm{u} 0} \times\left[\delta_{\mathrm{rem}}+\left(1-\delta_{\mathrm{rem}}\right) e^{-3 \xi / \xi_{95}}\right]
$$

In this expression the bracketed term defines the degree of softening as a function of the accumulated plastic shear strain, $\xi$. The parameter $\delta_{\text {rem }}$ is the ratio of the fully remoulded strength to the intact strength (i.e. the inverse of the soil sensitivity, $S_{t}$ ). The ductility parameter $\xi_{95}$ corresponds to the accumulated plastic shear strain at which the soil has undergone $95 \%$ of the reduction in strength due to remoulding. For typical soft marine clays, $S_{t}$ ranges from 2 to 5 and $\xi_{95}$ ranges from 10 to 50 (Randolph, 2004). 
1 For all of the SLA simulations described here, the pipe was pre-embedded into the soil by

$20.001 D$ and then pushed vertically under displacement control to the target embedment,

3 denoted Wini. Following installation, the pipe was subjected to displacement-controlled cyclic lateral movement under a prescribed vertical load $V$. This vertical load was always kept constant within a given lateral 'sweep' in one direction, but was sometimes varied from one sweep to the next.

\section{Details of simulations}

An appropriate suite of physical model tests for validating the performance of the SLA approach has been reported by Rismanchian (2014). These tests explored the cyclic lateral loading behaviour of a $20 \mathrm{~mm}$ diameter model pipe segment on soft kaolin clay, and were conducted in a geotechnical centrifuge at an acceleration level of $25 \mathrm{~g}$. The pipe and soil parameters from Rismanchian (2014), given at prototype scale, are summarised in Table 1. The roughness factor $\alpha$ was taken as zero since the model pipe was reported to be "smooth", but in reality there was probably some shear stress developed on the interface. The soil unit weight in Table 1 is the value measured at the soil surface. Although this value was reported (Rismanchian, 2014) to increase linearly with depth at a slight gradient of $0.1 \mathrm{kN} / \mathrm{m}^{3} / \mathrm{m}$, the surface value was used throughout the whole soil domain in the SLA simulations. The soil sensitivity in the centrifuge tests was in the range 2.8 to 5 . Therefore, the strain softening parameters used for the SLA simulations were $S_{t}=3.3$ and $\xi_{95}=10$, following the values adopted in previous numerical studies of similar problems (Wang et al., 2009; Chatterjee et al., 2012; Kong, 2015).

The focus of the SLA simulations was on modelling the first few cyclic lateral pipe movements in each of five selected tests performed by Rismanchian (2014). The details are given in Table 2, using the same test designations ( $A$ to $E$ ) as those used by Rismanchian. According to Rismanchian, Tests A-C were intended to mimic the behaviour of pipe sections close to the crown of a lateral buckle. Consequently, the vertical load $V$ applied to the pipe was varied during each test, to examine the effect of possible changes in contact force during the cyclic buckling process. These changes could arise as a result of three-dimensional (3D) bending 
effects (variation of elevation along the pipe within a buckle). Tests $D$ and $E$ represent hypothesised modes of behaviour relevant to pipe sections near the shoulder of a lateral buckle. A single large-amplitude lateral cycle is followed by a series of small-amplitude movements with a progressive offset in one direction, mimicking encroaching/retreating behaviour.

It should be noted that in the centrifuge model tests, the pipe was installed dynamically using small-amplitude lateral oscillations in conjunction with vertical insertion, while in the SLA simulations, a simple vertical penetration was employed as described above. Also, at the completion of the SLA simulations of Tests A-C, the pipe was displaced laterally by an additional $1 D$ in order to examine the merging of the active and dormant berms, and to study the trajectory of the pipe as it encountered the 'step' created by the previous cyclic reversal. This differs from the procedure adopted in the tests by Rismanchian (2014).

\section{Limitations of the present numerical model}

There are clearly some inherent simplifications made in the SLA model, which prevent certain aspects of pipeline behaviour from being captured realistically. For example, accurate modelling of a complete lateral buckle requires $3 \mathrm{D}$ analysis, since the vertical load on a particular pipe section depends heavily on the variation of elevation along the pipe within a buckle. As a result, the assumption of a constant vertical dead load on the plane-strain pipe section during an individual lateral sweep (although it varies for different sweeps) is somewhat idealised. However, studies of lateral pipe response under constant vertical load form an important basis for understanding of the global 3D behaviour of a buckling pipeline (e.g. Cheuk et al., 2007; White \& Dingle, 2011; Rismanchian, 2014). A significant improvement presented in this paper is the derivation of $\mathrm{V}-\mathrm{H}$ yield envelopes during the loading process, providing more insight into the possible pipe behaviour in the presence of a change in vertical load. More details regarding 3D modelling of lateral pipeline buckling are discussed in the Conclusions section.

Another limitation of the present SLA approach is that consolidation of the soil is not accounted for. During an individual lateral sweep, it is reasonable to assume undrained soil response (e.g. 
1 Wang et al., 2009; Chatterjee et al., 2012; Dutta et al., 2015). However, for cyclic loading cases

2 there could be long periods of rest between any two consecutive sweeps, depending on the

3 duration of operational start-ups and shut-downs. These rest periods may allow significant 4 consolidation, and therefore recovery of strength, of the soil to take place (Chatterjee et al., 5 2014). The inability of SLA to model consolidation could possibly lead to underestimation of the 6 breakout resistance at the beginning of a lateral movement that takes place after a rest period.

7 The assumption of a no-tension interface condition in the SLA simulations will also tend to 8 cause underestimation of the breakout resistance at the start of a sweep. The focus of this 9 paper, however, is on the modelling of very large lateral displacements applied over the course of several cyclic sweeps.

RESULTS

Figure 3 compares the key results from the five centrifuge model tests and the corresponding SLA simulations, presented as pipe invert trajectory and lateral load-displacement response. Very good overall agreement is observed across all five tests; the details of each one will be discussed in the following subsections. For all simulations the same representative soil strength profile, as given in Table 1, was adopted. Of course there are likely to be some local variations of the soil properties for each centrifuge test, and these variations will be one source of discrepancy between the experimental and numerical results.

The numerical resistance curves in Fig. 3 all show a characteristic 'sawtooth' pattern, which arises from the periodic interpolation used in the SLA process. These fluctuations are considered to be within an acceptable range, and are shown here to demonstrate the overall quality and stability of the SLA solutions. However, the parametric study results presented later were smoothed by taking the average of every ten consecutive data points, for the sake of clarity. 
Test A (Figs 3(a), 3(b), 4, 5, 6)

During sweep 1 of the centrifuge model test, the pipe breaks away from the soil almost immediately when loading begins, indicated by a peak and then a sharp reduction in lateral resistance (see Fig. 3(b)). As the sweep progresses, the pipe embedment and lateral soil resistance both gradually decrease, displaying typical 'light pipe' behaviour (Bruton et al., 2005; Chatterjee et al., 2012). A steady residual state is reached after about $3 D$ of lateral displacement. Immediately after the reversal of direction (the start of sweep 2) the pipe ploughs deeper into the soil (see Fig. 3(a)), reaching an essentially steady state after less than $1 D$ of backward movement. Similar observations can be made for sweep 3, though the reduction in $V$ from $2.05 \mathrm{kN} / \mathrm{m}$ to $1.25 \mathrm{kN} / \mathrm{m}$ (see Table 2) means that the pipe scrapes a smaller thickness of soil and encounters less lateral resistance than it did in the first two sweeps. It should also be noted that in the centrifuge model test there was a period of consolidation (6.5 days at prototype scale) before this loading phase commenced. As the lateral displacement approaches $u=8 D$ in sweep 3, the soil resistance increases sharply as the pipe nears the dormant berm (and the step in the intact soil surface) left by sweep 1.

From Figs 3(a) and 3(b) it is clear that the SLA simulation produces a very good representation of the pipe-soil response measured in the centrifuge model test, though the lateral resistance on the first reversal (sweep 2) is moderately underestimated. In Fig. 3(b) it can be noted that the experimental load-displacement results exhibit a small peak at the beginning of sweep 3 , which is not observed in the numerical calculation. As there is no similar response at the start of sweep 2, it is likely that the peak is attributable to soil consolidation during the rest period prior to sweep 3 (see Table 2), leading to enhancement of the local undrained strength. During consolidation, the pipe would also be expected to gain improved adhesion to the disturbed soil in the berm, allowing pore water suctions to be sustained for longer during the subsequent sweep. The consolidation behaviour and any breakout suction are not captured by the SLA method used here. 
1 From the output of the SLA simulation it is possible to interrogate various details of the pipe-soil

2 interaction. This is illustrated in Fig. 4, where the evolution of the soil surface profile, mesh and

3 normalised shear strain rate are presented. To highlight the field variables, only the domain

4 near the deforming region is shown. The shear strain rates $\dot{\gamma}$ are normalised using the pipe diameter $D$ and pipe loading speed $v_{p}$. Typical failure mechanisms for a light pipe are shown in Figs 4(b) and 4(c), where the slip-lines at the base of the active berm lie wholly in the horizontal direction (cf. Dingle et al., 2008; Wang et al., 2009; Chatterjee et al., 2012). Figures 4(d) and 4(e) show in detail how the active berm is predicted to merge with the dormant berm deposited at the end of sweep 1. The calculated velocity field in the soil around the pipe is also illustrated. As the pipe moves through the dormant berm and scrapes across the step in the intact soil surface beneath, there is considerable rebound in the pipe invert elevation (Fig. 3(a)) and a drop in the lateral resistance (Fig. 3(b)).

Figure 5 shows the distribution of the softening factor (the bracketed term in equation (1)) near the end of the SLA simulation. Three different soil berms are observed: the current active one, and dormant berms to the left and right. The majority of the soil within the berms appears to be almost fully remoulded (softening factor $=1 / 3.3=0.3$ ), while the soil between the berms is far less softened. The depth of penetration of shearing is limited to a shallow band beneath the freshly-scraped soil surface.

Another interesting detail of the pipe-soil interaction that can be studied using SLA is the evolution of the $V-H$ failure envelope for the pipe. This is illustrated in Fig. 6 for sweeps 1 and 3. The failure envelopes are obtained by subjecting the pipe to displacement-controlled probes in all directions, at an interval of $1^{\circ}$. The clear advantage of the numerical approach is the ability to break into the simulation at any point and explore in more detail the full range of possible loading paths at that point. The results obtained are consistent with the load-displacement curve in Fig. 3(b), and provide useful information on likely pipe behaviour if the inputs are changed (e.g. a change in vertical contact force or direction of pipe movement). All of the $\mathrm{V}-\mathrm{H}$ failure envelopes show a trend of rotation towards the sweep direction (i.e. to the right) since the active berm lies on the right of the pipe during these sweeps. In these envelopes $H$ is never 
negative, which shows that moving the pipe 'backwards' does not necessarily need a backwards force. This counterintuitive situation arises because a stable incremental backwards motion requires a force in the forwards direction to prevent the remoulded soil berm from collapsing. Figure $6(\mathrm{a})$ shows that, beyond a lateral displacement of $1 D$ during sweep 1 , the failure envelopes are all very similar in both size and shape, indicating steady residual behaviour. Figure $6(\mathrm{~b})$ shows the expansion and shrinkage of the failure envelope near the end of sweep 3, when the pipe encounters a dormant berm and scrapes over it (see Figs 4(d) and $4(e))$

\section{Test B (Figs 3(c), 3(d), 7, 8)}

For this test there is again good general agreement between the centrifuge model test and its SLA simulation (see Figs 3(c) and 3(d)). The trajectory from SLA overestimates the pipe embedment by about $0.1 D$ during sweep 2, conducted with $V=4.05 \mathrm{kN} / \mathrm{m}$, but it correctly predicts that only a thin layer of soil is scraped away during sweep 3 , for which $V$ is substantially reduced to $1.25 \mathrm{kN} / \mathrm{m}$ (see Table 2). The peak in lateral resistance measured at the start of sweep 1 in the centrifuge test is not captured by the SLA modelling, as this is principally due to suction developed at the rear of the pipe. As in Test A, the initial peak in resistance for sweep 3 following a period of consolidation is also not captured by the numerical analysis.

In the three sweeps plotted in Fig. 3(d), the lateral resistance stabilises after about $1 D$ of movement, indicating that an essentially steady residual state has been achieved (Bruton et al., 2005; Dingle et al., 2008; White \& Dingle, 2011). Both the experimental and numerical results show sharp increases in lateral resistance at the end of sweeps 2 and 3 , which are due to the pipe pushing into and over the previously formed berms. In the SLA simulation, at the end of sweep 3 , the pipe is pushed $1 D$ past the previous lateral extremity. Here the soil resistance firstly increases and then reduces as the pipe (now weighing only $1.25 \mathrm{kN} / \mathrm{m}$ ) rides up and into the dormant berm. Such behaviour is not observed in the centrifuge model test as the pipe was not displaced this far. Some substantial oscillations are apparent in the numerical resistance curve at the beginning of sweep 2, when the pipe moves away from the dormant berm. The 
sudden separation leads to a significant reduction in the vertical bearing capacity of the pipe, which at some increments could be smaller than the applied vertical dead load. To avoid unstable collapse of the model under the vertical dead load, the pipe was forced to move under pure displacement control for these increments, leading to different failure mechanisms. Slope instability of the dormant berm just after its separation from the pipe may also have contributed to this unstable collapse behaviour (Kong et al., 2016).

Figure 7 shows normalised shear strain rates and velocity fields for selected instants of the cyclic lateral displacement sequence. This shows similar behaviour to Test A (Fig. 4) but with much bigger berms being formed by the heavier pipe during the first two sweeps ( $V=4.05 \mathrm{kN} / \mathrm{m}$ in Test B compared with $2.05 \mathrm{kN} / \mathrm{m}$ in Test A). At the start of sweep 2, as the pipe is moved away from the berm (see Fig. 7(b)), the heavily softened soil in the berm is unable to support its own weight, resulting in an additional driving force on the pipe. This significantly decreases the net lateral resistance as the pipe motion is reversed. Figures $7(\mathrm{~d})$ and $7(\mathrm{e})$ illustrate the complex process of the pipe approaching the dormant berm and then pushing up and into it.

Finally, Fig. 8 illustrates the softening factor throughout the soil at the end of sweep 2. The soil within the berms shows different degrees of softening, with some being fully remoulded and some undisturbed. However, the most intense shearing (see the slip-lines in Figs 7(a) and 7(c)) always takes place in the weakest region of the berm, and in a simpler (non-sequential) numerical analysis it would be reasonable to assume fully remoulded strength throughout the whole berm when evaluating the lateral resistance, as proposed by Rismanchian (2014).

\section{Test C (Figs 3(e), 3(f), 9)}

This test is slightly different from, and more complicated than, Tests A and B, since three different vertical loads are used for the three sweeps (see Table 2). However, highly satisfactory SLA predictions can still be observed for both the invert trajectory and the lateral resistance, as shown in Figs 3(e) and 3(f) respectively. In this test, as noted in Table 2, the pipe was rested to allow for consolidation prior to each sweep. The peaks in lateral resistance observed at the 
beginning of each sweep in the experiment are therefore not reflected in the numerical results obtained using SLA.

Figure 9 shows the normalised shear strain rates and velocity field in the final stages of the SLA simulation, where the active berm on sweep 3 is merging with the dormant berm deposited at the end of sweep 1. In a similar way to Tests A and B, it can be seen that the soil regions experiencing intense shearing are successfully discretised with smaller elements.

\section{Test $D$ (Figs 3(g), 3(h), 10)}

Unlike Tests $A$ to $C$, Figs $3(\mathrm{~g})$ and $3(\mathrm{~h})$ show that for Test $\mathrm{D}$, the centrifuge model test results exhibit deeper pipe embedment and greater lateral resistance than the SLA prediction for the whole of the first loading cycle (sweeps 1 and 2). During the cyclic encroaching and retreating phase of the test (sweeps 3 to 12) the agreement between the experimental and numerical trajectories gradually improves, but the lateral resistance is still overpredicted by the SLA simulation. This could be because the soil strength profile adopted in the SLA modelling, which is consistent across all analyses, is stronger than the actual soil strength at the location of Test D in the centrifuge strongbox. It is also noteworthy that of the five tests simulated, this one uses the smallest vertical load during sweep $1(V=1.35 \mathrm{kN} / \mathrm{m}$, see Table 2). The tendency of the light pipe to rise during the initial lateral displacement means that both the experimental and numerical results are sensitive to the undrained strength near the mudline (the upper few millimetres of soil at model scale in the centrifuge). It is possible to obtain a better match with the experimental data by tuning the SLA strength profile accordingly, but this is not explored further here, as it does not impact on the main conclusions drawn from this paper. The overall predicted behaviour of the pipe in both trajectory space and lateral load-displacement space is satisfactorily consistent with that observed in the centrifuge test.

Figure 10 shows two snapshots of the soil surface profile and the distribution of shear strain rate obtained from the SLA simulation during the last set of cyclic sweeps. The size of the berm is extremely small in the retreating (left) direction on account of the low pipe weight, but is 
significantly larger in the encroaching (right) direction due to the merging of an active berm into a dormant berm during each sweep.

\section{Test E (Figs 3(i), 3(j), 11, 12, 13)}

There is again very satisfactory agreement between the experimental and numerical results for this test, despite the heavy pipe weight ( $V=5.35 \mathrm{kN} / \mathrm{m}$ throughout) and the complex sequence of cyclic lateral displacements applied to the pipe. In the final phase of the test (sweeps 3 to 12) the SLA simulation provides a moderate underestimate of the lateral resistance for the encroaching stages, and an overestimate for the retreating stages (see Fig. 3(j)). Figure 11 shows the soil surface profile and shear strain rate distribution when the pipe reaches the right extremity of movement in sweeps 3,5 and 11 . This clearly illustrates the various soil failure mechanisms at play, and shows how soil berms can lead to both driving and resistive forces on the pipe. In this particular scenario a larger force is needed to displace the pipe to the left than to the right. This behaviour ceases once the pipe has been driven sufficiently far towards the right (see Fig. 11(c)) to allow the pipe to break away from the berm on the left.

Figure 12 shows the distribution of the softening factor (see equation (1)) at the end of sweep 11, illustrating a complex set of conditions within the soil mass. Interestingly, the region with the greatest strength reduction is in the berm to the left of the pipe, rather than the one created by the rightward encroaching motions. This is explained by the failure mechanisms shown in Figs $11(a)$ and 11 (b), where shearing deformations take place in the berm on the left side of the pipe even when the pipe is moving to the right. Figure 13 presents $V$ - $H$ failure envelopes for the pipe at the extremities during the encroaching/retreating cycles. In a similar way to the behaviour illustrated for Test A in Fig. 6, the envelopes in Fig. 13(a) show a trend of rotating towards the direction of pipe movement (leftward). However different behaviour is observed in Fig. 13(b), where some failure envelopes still show deviation towards the left, even though the pipe is at the extremity of its rightward movement. This behaviour is consistent with the failure mechanisms shown in Fig. 11. 


\section{DISCUSSION}

Across Tests $\mathrm{A}, \mathrm{B}$ and $\mathrm{C}$, the vertical loads applied during sweeps 1 and 2 (denoted $V_{1}$ and $V_{2}$ hereafter) spanned a relatively wide range, but always reverted to a common value during sweep $3\left(V_{3}=1.25 \mathrm{kN} / \mathrm{m}\right.$, see Table 2). Figure 14 shows a comparison of the SLA simulations of sweep 3 in Tests A, B and C. The displacement trajectories in Fig. 14(a) differ considerably, indicating a significant influence of the previous vertical loading history. However, all of the lateral load-displacement curves in Fig. 14(b) agree very well during the steady residual stage between $u=2 D$ and $5 D$. This is expected because all three pipes have the same weight during sweep 3, so each one scrapes away approximately the same thickness of soil from the surface profile left behind by sweep 2 . The most pronounced difference can be found near the right extremity of each simulation, where a peak in lateral resistance is reached as the pipe pushes through the dormant berm and encounters the step in the intact soil surface beneath. This peak resistance increases considerably with the magnitude of the previously applied vertical load $V_{1}$, since this determines the size of the dormant berm (and the height of the step) created at the end of sweep 1.

Having validated the SLA approach through the extensive comparisons with experimental data presented above, numerical parametric studies can now be used to perform further systematic investigations of pipe-soil interaction during cyclic lateral displacements under varying vertical loads. Figure 15 shows the results of two hypothetical 'tests' using the vertical loads from Test A and the lateral displacement sequence from Test $B$, but with two different values of the initial pipe embedment $\left(W_{\text {ini }}=0.15 D\right.$ and $0.45 D$ ). Following initial breakout, and beyond about $u=1.5 D$, the two trajectory curves and the two resistance curves are very similar. There are differences in the early part of sweep 1, with the initially deeper pipe reaching a slightly higher elevation and encountering more lateral resistance. The resistances obtained from both simulations are extremely close during the steady residual stages of sweeps 2 and 3 in the range $u=2 D$ to $6 D$. Very close agreement between the two cases can be noted even in the initial part of sweep 2, where rapid changes occur in both pipe embedment and lateral soil resistance. In general these SLA simulations confirm that, as expected, the influence of $W_{\text {ini }}$ on 
lateral resistance diminishes with the distance travelled by the pipe. The observations here are consistent with the parametric study of the monotonic lateral loading behaviour of a pipe reported by Kong (2015), where the data covered a wide range of Wini and V. However, the present results differ from those presented by Chatterjee et al. (2012), where a weak dependence of residual lateral resistance on initial embedment was found. A possible reason for this difference is that the lateral displacement in Chatterjee et al. (2012) was $3 D$, which may not be large enough to develop a steady residual state. As shown in Fig. 15(b), a moderate difference in soil resistance can still be observed up to a lateral displacement of $3 D$, but the difference diminishes with further displacement. Another reason could be the inability of SLA to model the elastic response of the soil, which is captured in the RITSS method employed by Chatterjee et al. (2012).

Figure 16 presents SLA simulations of four further hypothetical 'tests' that illustrate how the vertical load applied during sweeps 1 and 2 affects the lateral soil resistance developed during sweep 3. In all cases the initial embedment was $W_{\text {ini }}=0.45 D$ and the lateral displacement sequence was the same as that used for the SLA simulation of Test B, including the additional $1 D$ of lateral displacement into the dormant berm at the end of sweep 3 . The vertical loads chosen for the first two sweeps were $V_{1}=V_{2}=1.25,2.05,3.00$ and $4.05 \mathrm{kN} / \mathrm{m}$, as these were values adopted in the centrifuge tests of Rismanchian (2014). Two different cases were then considered by choosing $V_{3}$ to be the smallest or largest value of $V$ used during sweeps 1 and 2, resulting in over-penetrated and normally-penetrated conditions respectively. Figure 16(a) shows the results for the over-penetrated case. The soil resistances during the steady residual stage are very close for all four curves, indicating limited influence of the previous vertical load on the current vertical load-displacement response, even considering that the current pipe embedment varies over a wide range. As the pipe approaches and pushes into the dormant berm, the corresponding peak and residual resistances both increase with the vertical load used during sweeps 1 and 2, owing to the larger size of berm and step ahead of the pipe. Similar observations can be made for the normally-penetrated case, shown in Fig. 16(b). However, the heavy normally-penetrated pipe does not reach a steady residual state during sweep 3 in the 
same way as the light over-penetrated pipe, and moderate differences in the resistance curves are observed.

\section{CONCLUSIONS}

This paper has presented a numerical study using sequential limit analysis (SLA) to simulate the behaviour of a plane-strain pipe segment undergoing large-amplitude cyclic lateral displacements on undrained clay. The work is relevant to untrenched subsea pipelines that operate under HPHT conditions and are designed to undergo controlled lateral buckling. Using SLA, the progressive evolution of the soil surface profile, including the formation of active and dormant berms, was modelled directly, and the effect of strain softening was accounted for using the approach suggested by Einav \& Randolph (2005). Validation of SLA predictions against centrifuge model test results (Rismanchian, 2014) was performed for five complex loading cases. Various aspects of the pipe-soil interaction were explored in detail using information derived from the numerical modelling, including an exposition of soil failure mechanisms and $\mathrm{V}-\mathrm{H}$ failure envelopes at salient points in the cyclic analyses. A brief numerical study was also presented to provide insight into the influence of the initial vertical loading history on the subsequent pipe behaviour.

Despite the complex cyclic loading histories employed in the centrifuge model tests, including step changes in vertical load from one lateral sweep to the next, the numerical results obtained using SLA showed good agreement with the corresponding experiments, in terms of both the pipe invert trajectory and the lateral load-displacement response. The prediction of the steady residual response of light pipes at large lateral displacements was very satisfactory. The increase in lateral soil resistance as the pipe approaches a dormant berm, and the drop in resistance when the pipe moves away from it or scrapes over it, was also accurately captured. However, the SLA approach is not currently able to model the effects of suction at the pipe-soil interface or soil strength enhancement due to consolidation. This was highlighted by a mismatch between the numerical predictions and the experimental results for the peak in 
resistance occurring during the initial lateral breakout, and during lateral displacement reversals that were preceded by a rest period allowing consolidation.

The SLA simulations have illustrated that as a laterally buckling pipe scrapes across the soil surface to develop a berm, the soil within the berm can experience severe remoulding. The overall degree of remoulding decreases considerably as the berm size increases, and this in turn is strongly dependent on the vertical dead load applied to the pipe. A light pipe scrapes a shallow layer of soil and develops a small berm that is fully remoulded, while a heavy pipe creates a larger berm containing soil regions with different degrees of remoulding, some material being fully remoulded and some undisturbed. The most intense soil shearing is found to take place in the weakest region of a berm, and in a simpler (non-sequential) analysis it is recommended to assume fully remoulded undrained strength throughout all berms when evaluating the lateral resistance.

The numerical analyses performed for this paper involved cumulative cyclic lateral displacements of up to 23 pipe diameters. Each analysis took less than a day to complete on a 4-core desktop computer with 4 GB RAM. The efficiency of the SLA method for this type of problem suggests two possible directions for future investigations. One direction might involve conducting a large set of parametric studies for the extraction of lateral resistance and full $\mathrm{V}-\mathrm{H}$ failure envelopes at different loading stages, for pipes of different weights and initial overpenetration ratios. This could inform the development of a macro-element plasticity model for incorporation into 3D pipe-soil interaction analyses. A second direction might be the development of a 3D numerical model for a laterally buckling pipeline incorporating a number of 2D soil 'slices', as suggested by Martin et al. (2013), but using SLA rather than CEL finite element analysis within each slice. At present it is not practical to carry out fully $3 \mathrm{D}$ numerical simulations of lateral pipe-soil interaction for routine design (Konuk \& Yu, 2007), but the suggested alternative methods based on plane-strain SLA could potentially contribute to better understanding and improved prediction of the lateral buckling behaviour of offshore pipelines. 


\section{REFERENCES}

Almeida, M. S. S., Costa, A. M., Amaral, C. S., Benjamin, A. C., Noronha Jr, D. B., Futai, M. M. \& Mello, J. R. (2001). Pipeline failure on very soft clay. Proceedings of the 3rd International Conference on Soft Soil Engineering, Hong Kong, 131-138.

Aubeny, C. P., Shi, H. \& Murff, J. D. (2005). Collapse loads for a cylinder embedded in trench in cohesive soil. International Journal of Geomechanics 5, No. 4, 320-325.

Bruton, D., Carr, M., Crawford, M. \& Poiate, E. (2005). The safe design of hot on-bottom pipelines with lateral buckling using the design guidance developed by the SAFEBUCK Joint Industry Project. Deep Offshore Technology Conference, Vitoria, Espirito Santo, Brazil.

Bruton, D., Carr, M. \& White, D. J. (2007). The influence of pipe-soil interaction on lateral buckling and walking of pipelines: the SAFEBUCK JIP. Proceedings of the 6th International Conference on Offshore Site Investigation and Geotechnics, London, 133-150.

Chatterjee, S. (2012). Numerical modelling of pipe-soil interactions. PhD thesis, University of Western Australia.

Chatterjee, S., White, D. J. \& Randolph, M. F. (2012). Numerical simulations of pipe-soil interaction during large lateral movements on clay. Géotechnique 62, No. 8, 693-705.

Chatterjee, S., Gourvenec, S. \& White, D. J. (2014). Assessment of the consolidated breakout response of partially embedded subsea pipelines. Géotechnique 64, No. 5, 391-399.

Cheuk, C. Y. (2005). Soil-pipeline interaction at the seabed. PhD thesis, University of Cambridge.

Cheuk, C. Y. \& White, D. J. (2009). Modelling the dynamic embedment of seabed pipelines. Géotechnique 61, No. 1, 39-57.

Cheuk, C. Y., White, D. J. \& Bolton, M. D. (2007). Large-scale modelling of soil-pipe interaction during large amplitude cyclic movements of partially embedded pipelines. Canadian Geotechnical Journal 44, 977-996.

Dingle, H. R. C., White, D. J. \& Gaudin, C. (2008). Mechanisms of pipe embedment and lateral breakout on soft clay. Canadian Geotechnical Journal 45, 636-652.

Dutta, S., Hawlader, B. \& Rhillips, R. (2015). Finite element modeling of partially embedded pipelines in clay seabed using Coupled Eulerian-Lagrangian method. Canadian Geotechnical Journal 52, 58-72.

Einav, I. \& Randolph, M. F. (2005). Combining upper bound and strain path methods for evaluating penetration resistance. International Journal for Numerical Methods in Engineering 63, No. 14, 1991-2016.

Hesar, M. (2004). Pipeline-seabed interaction in soft clay. Proceedings of the 23rd International Conference on Offshore Mechanics and Arctic Engineering, Vancouver, Paper No. OMAE2004-51425, 225-233.

Hu, Y. \& Randolph, M. F. (1998). A practical numerical approach for large deformation problems in soil. International Journal for Numerical and Analytical Methods in Geomechanics 22, 327-350. 
Hwan, C. L. (1997). An upper bound finite element procedure for solving large plane strain deformation. International Journal for Numerical Methods in Engineering 40, 1909-1922.

Kong, D. (2015). Large displacement numerical analysis of offshore pipe-soil interaction on clay. DPhil thesis, University of Oxford.

Kong, D., Martin, C. M. \& Byrne, B. W. (2016). Modelling large plastic deformations of cohesive soils using sequential limit analysis. International Journal for Numerical and Analytical Methods in Geomechanics, under review.

Konuk, I. \& Yu, S. (2007). Continuum FE modelling of lateral buckling: study of soil effects. Proceedings of the 26th International Conference on Offshore Mechanics and Arctic Engineering, San Diego, Paper No. OMAE2007-29376, 347-354.

Macaro, G. (2015). Distinct element modelling of pipe-soil interaction for offshore pipelines on granular soils. DPhil thesis, University of Oxford.

Makrodimopoulos, A. \& Martin, C. M. (2006). Lower bound limit analysis of cohesive frictional materials using second-order cone programming. International Journal for Numerical Methods in Engineering 66, No. 4, 604-634.

Makrodimopoulos, A. \& Martin, C. M. (2007). Upper bound limit analysis using simplex strain elements and second-order cone programming. International Journal for Numerical and Analytical Methods in Geomechanics 31, 835-865.

Makrodimopoulos, A. \& Martin, C. M. (2008). Upper bound limit analysis using discontinuous quadratic displacement fields. Communications in Numerical Methods in Engineering 24, 911-927.

Martin, C. M. (2011). The use of adaptive finite-element limit analysis to reveal slip-line fields. Géotechnique Letters 1, 23-29.

Martin, C. M., Kong, D. \& Byrne, B. W. (2013). 3D analysis of transverse pipe-soil interaction using 2D soil slices. Géotechnique Letters 3, 119-123.

Martin, C. M. \& Randolph, M. F. (2006). Upper bound analysis of lateral pile capacity in cohesive soil. Géotechnique 56, No. 2, 141-145.

MOSEK ApS (2010). The MOSEK optimization tools manual, version 5.

Murff, J. D., Wanger, D. A. \& Randolph, M. F. (1989). Pipe penetration in cohesive soil. Géotechnique 39, No. 2, 213-229.

Raithatha, A. \& Duncan, S. R. (2008). Rigid plastic model of incremental sheet deformation using second-order cone programming. International Journal for Numerical Methods in Engineering 78, 955-979.

Randolph, M. F. (2004). Characterization of soft sediments for offshore applications. Proceedings of the 2nd International Conference on Site Characterization, Porto, 209-231.

Randolph, M. F. \& Houlsby, G. T. (1984). The limiting pressure on a circular pile loaded laterally in cohesive soil. Géotechnique 34, No. 4, 613-623.

Randolph, M. F. \& White, D. J. (2008a). Pipeline embedment in deep water: processes and quantitative assessment. Proceedings of the Offshore Technology Conference, Houston, Paper No. OTC 19128. 
Randolph, M. F. \& White, D. J. (2008b). Upper-bound yield envelopes for pipelines at shallow embedment in clay. Géotechnique 58, No. 4, 297-301.

3 Rismanchian, A. (2014). Pipe-soil interaction during lateral buckling of marine pipelines. PhD 4 thesis, University of Western Australia.

5 Sabetamal, H. (2014). Finite element algorithms for dynamic analysis of geotechnical problems.

$6 \quad \mathrm{PhD}$ thesis, University of Newcastle, Australia.

7 Shewchuk, J. R. (2002). Delaunay refinement algorithms for triangular mesh generation. 8 Computational Geometry 47, 741-778.

9 Urthaler, Y., Watson, Ryan. \& Davis, J. (2012) Lateral buckling of deepwater pipelines in 10 operation. Proceedings of the 31st International Conference on Ocean, Offshore and Arctic 11 Engineering, Rio de Janeiro, Paper No. OMAE2012-83949, 783-792.

12 Wang, D., White, D. J. \& Randolph, M. F. (2009). Large-deformation finite element analysis of 13 pipe penetration and large-amplitude lateral displacement. Canadian Geotechnical Journal $14 \quad 47,842-856$.

15 White, D. J. \& Dingle, H. R. C. (2011). The mechanism of steady 'friction' between seabed pipelines and clay soils. Géotechnique 61, No. 12, 1035-1041.

17 Yang, W. H. (1993). Large deformation of structures by sequential limit analysis. International Journal of Solids and Structures 30, No. 7, 1001-1013. 


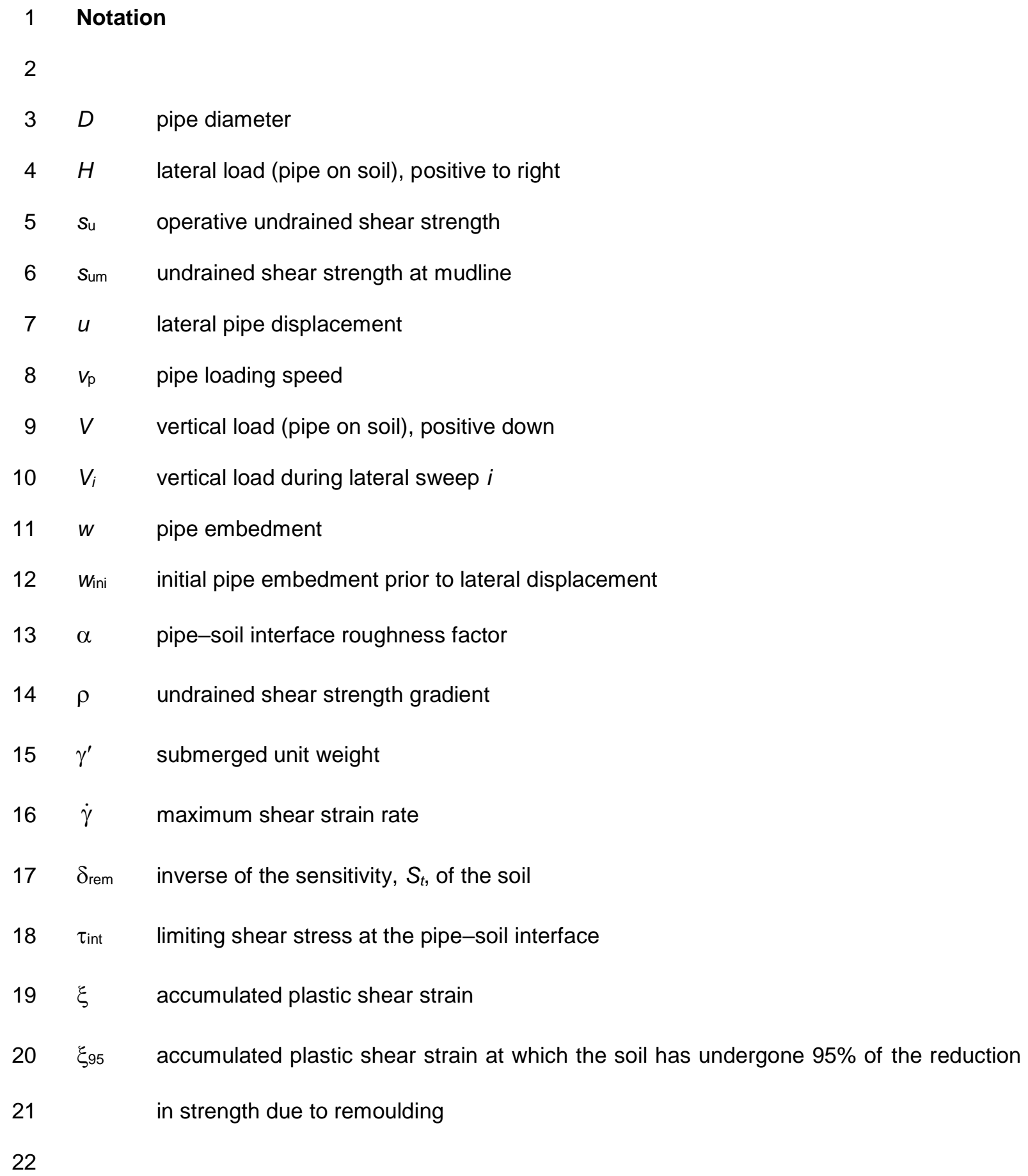




\section{Table captions}

2

3 Table 1. Centrifuge model test parameters (prototype scale) from Rismanchian (2014)

4

5 Table 2. Summary of first few lateral sweeps in centrifuge model tests of Rismanchian (2014)

6

7 


\section{Figure captions}

Figure 1. Overall scheme of the SLA approach (Kong et al., 2016)

Figure 2. Typical initial configuration of SLA model for vertical penetration and cyclic lateral loading of pipe (model shown is for Test A)

Figure 3. Pipe invert trajectory and lateral resistance during cyclic lateral loading: (a)-(b) Test A; (c)-(d) Test B; (e)-(f) Test C; (g)-(h) Test D; (i)-(j) Test E

Figure 4. Normalised shear strain rate $\left(\dot{\gamma} D / v_{\mathrm{p}}\right)$ and velocity field in Test A: (a)-(e) correspond to points $A 1-A 5$ in Fig. $3(b)$

Figure 5. Softening factor for soil strength in Test A: sweep 3, $u / D=7.5$

Figure 6. Evolution of $V-H$ failure envelope in Test A: (a) sweep 1; (b) sweep 3

Figure 7. Normalised shear strain rate $\left(\dot{\gamma} D / v_{\mathrm{p}}\right)$ and velocity field in Test B: (a)-(e) correspond to points B1-B5 in Fig. 3(d)

Figure 8. Softening factor for soil strength in Test B: sweep 2, $u / D=1$

Figure 9. Normalised shear strain rate $\left(\dot{\gamma} D / v_{\mathrm{p}}\right)$ and velocity field in Test C: (a) and (b) correspond to points $\mathrm{C} 1$ and $\mathrm{C} 2$ in Fig. 3(f)

Figure 10. Normalised shear strain rate $\left(\dot{\gamma} D / v_{\mathrm{p}}\right)$ in Test D: (a) and (b) correspond to points $\mathrm{D} 1$ and D2 in Fig. 3(g) 
1 Figure 11. Normalised shear strain rate $\left(\dot{\gamma} D / v_{\mathrm{p}}\right)$ and velocity field in Test E: (a)-(c) correspond

2 to the right extremities during sweeps 3, 5 and 11, points E1-E3 in Fig. 3(i)

3

4 Figure 12. Softening factor for soil strength in Test E: sweep 11, right extremity

5

6 Figure 13. Evolution of $V-H$ failure envelope in Test $E$ during sweeps 3-11: (a) left extremities;

7 (b) right extremities

8

9 Figure 14. Comparison of SLA simulations of Tests A, B and C during sweep 3: (a) pipe invert trajectory; (b) lateral resistance

Figure 15. Effect of initial embedment on cyclic lateral loading response: (a) pipe invert trajectory; (b) lateral resistance. For all analyses $V_{1}=V_{2}=2.05 \mathrm{kN} / \mathrm{m}, V_{3}=1.25 \mathrm{kN} / \mathrm{m}$

Figure 16. Effect of previous vertical loading history on lateral resistance during sweep 3: (a) over-penetrated pipe; (b) normally-penetrated pipe. For all analyses $w_{\text {ini }} / D=0.45$ 


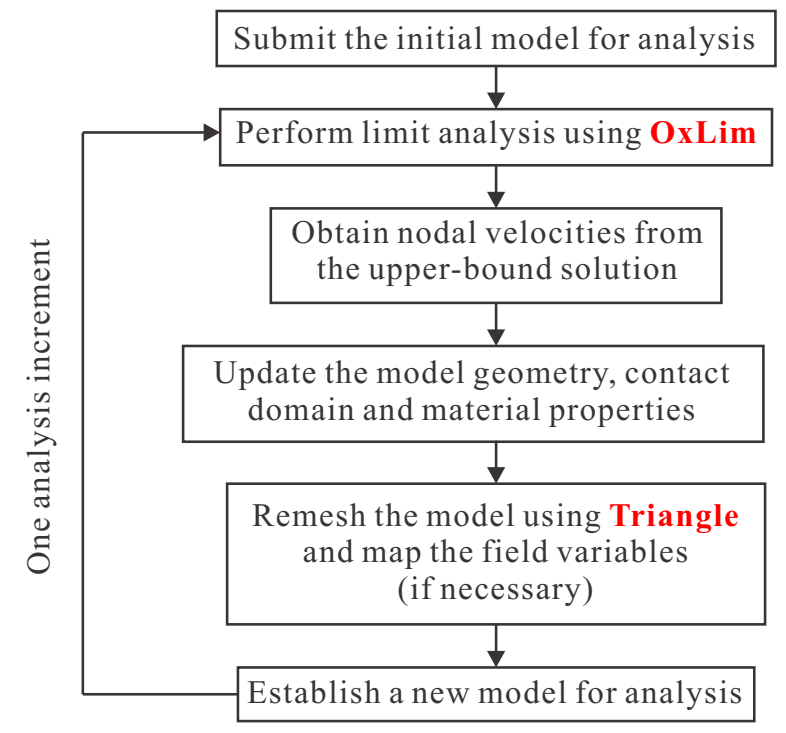

Fig. 1. Overall scheme of the SLA approach (Kong et al., 2016)

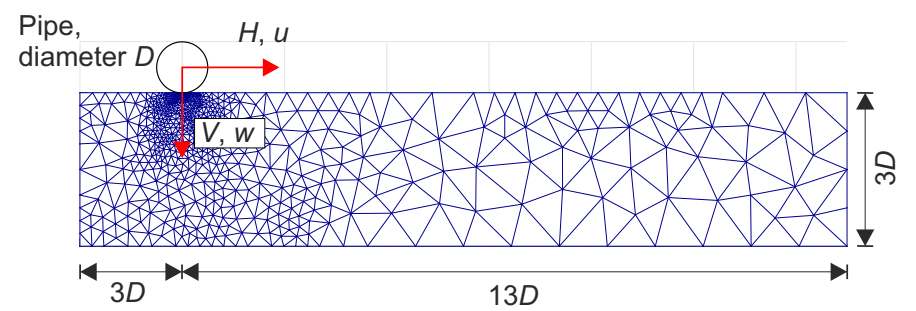

Fig. 2. Typical initial configuration of SLA model for vertical penetration and cyclic lateral loading of pipe (model shown is for Test A) 


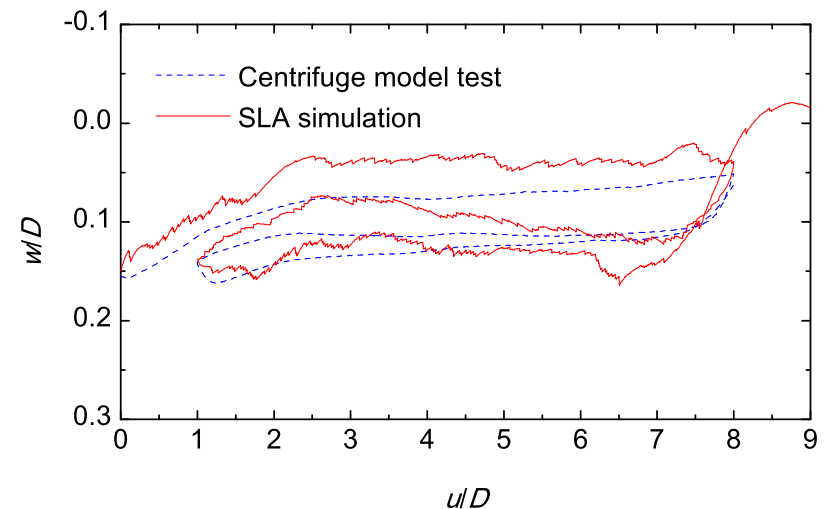

(a)

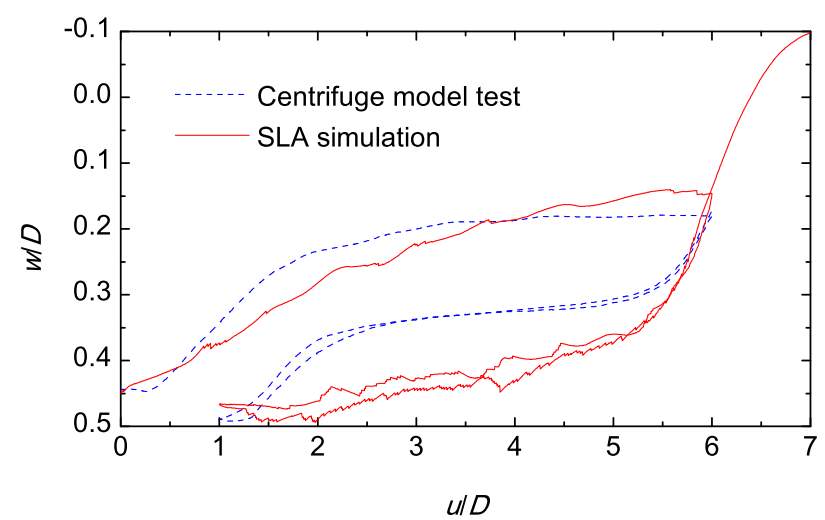

(c)

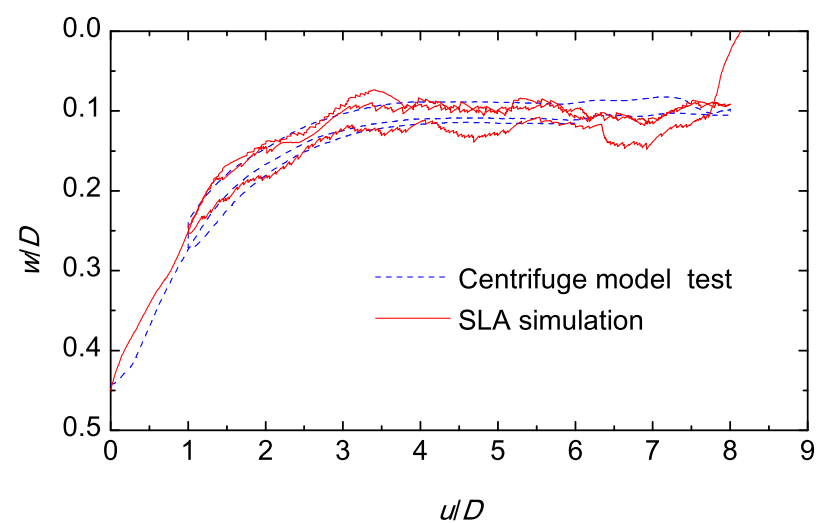

(e)

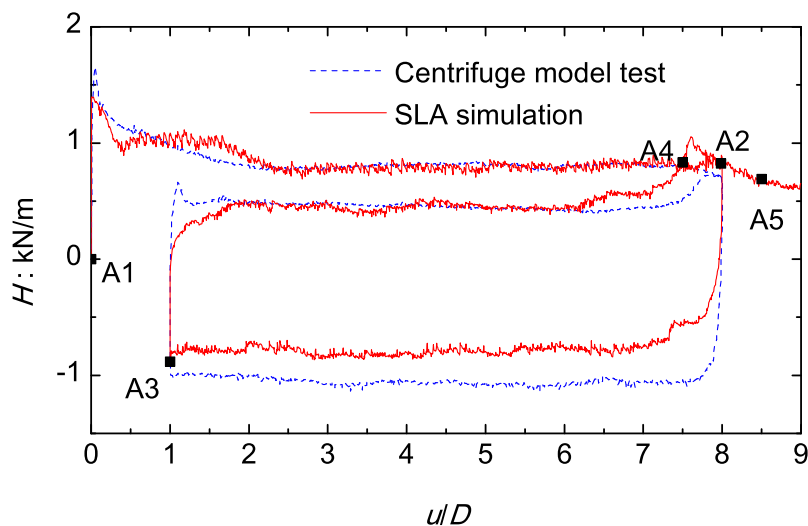

(b)

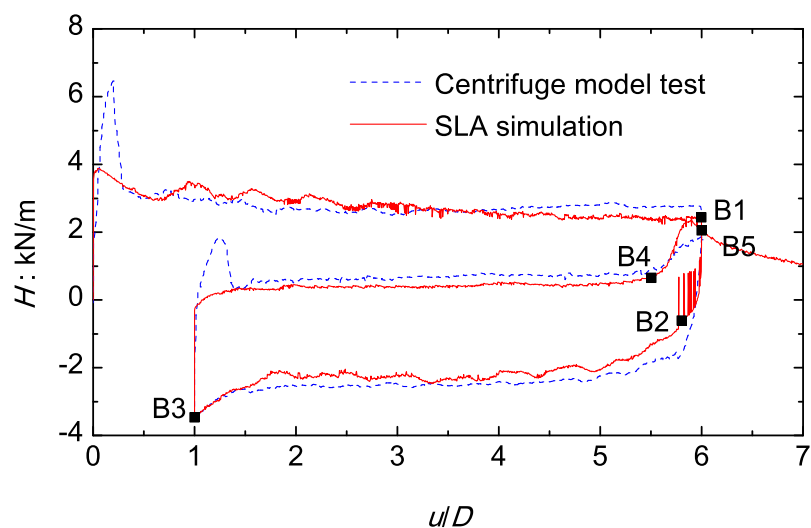

(d)

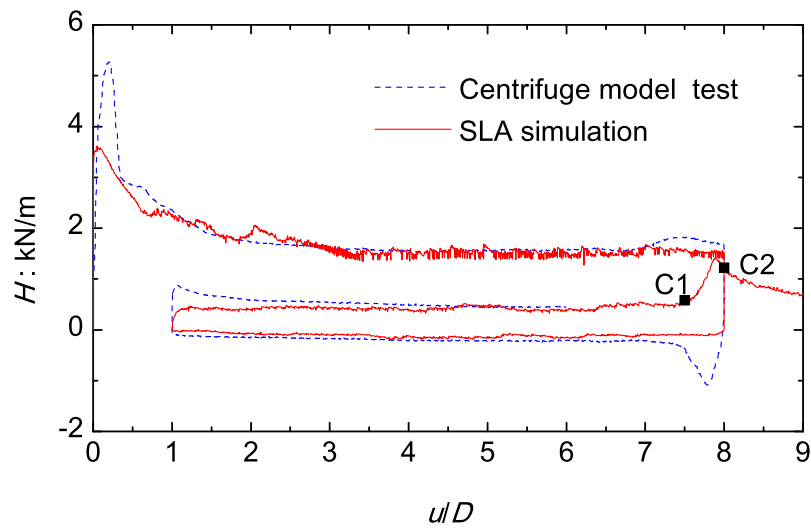

(f) 

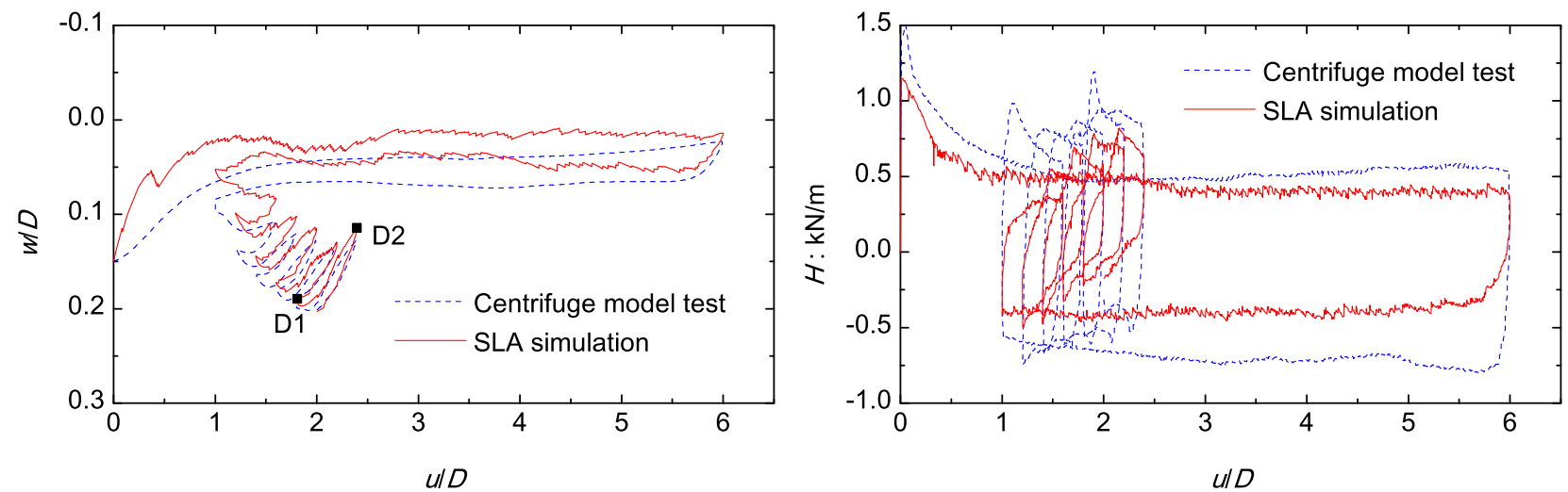

(g)

(h)
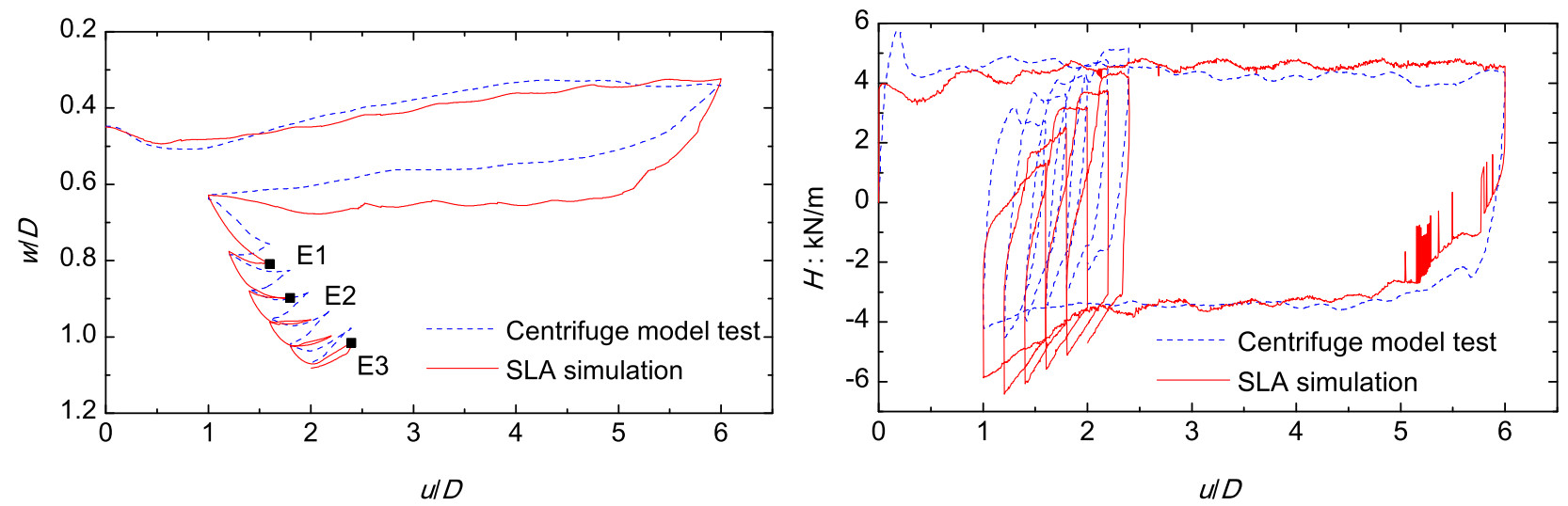

(i)

(j)

Fig. 3. Pipe invert trajectory and lateral resistance during cyclic lateral loading: (a)-(b) Test A; (c)-(d) Test B; (e)-(f) Test C; (g)-(h) Test D; (i)-(j) Test E 


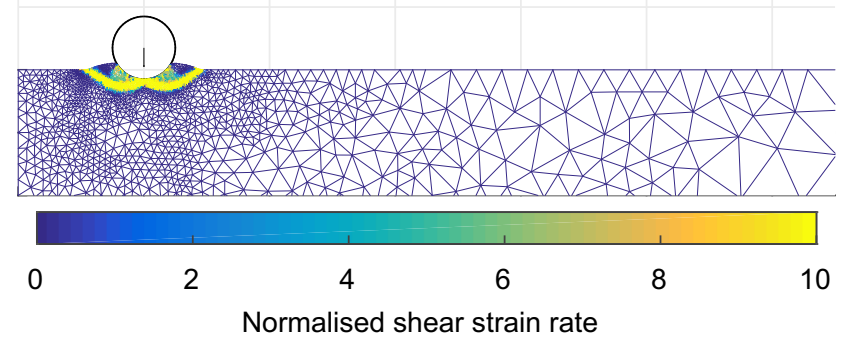

(a)

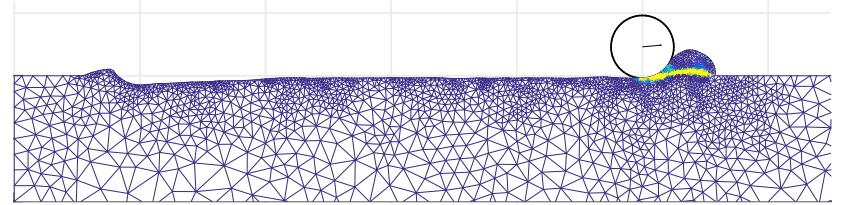

(b)

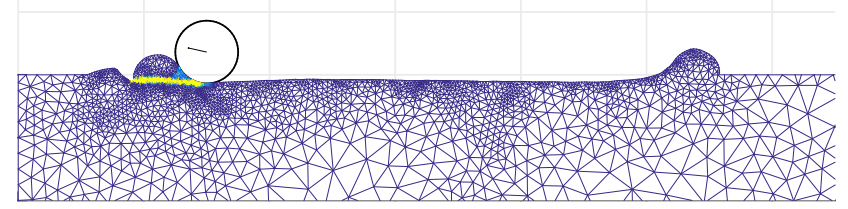

(c)

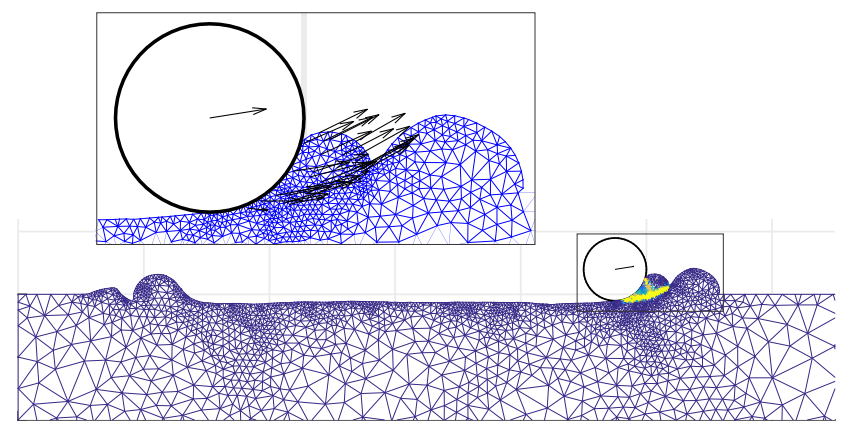

(d)

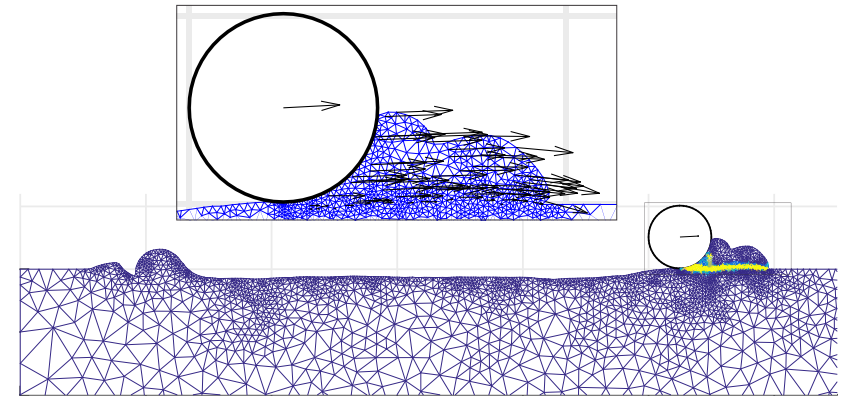

(e)

Fig. 4. Normalised shear strain rate $\left(\dot{\gamma} D / v_{p}\right)$ and velocity field in Test A: (a)-(e) correspond to points A1-A5 in Fig. 3(b)

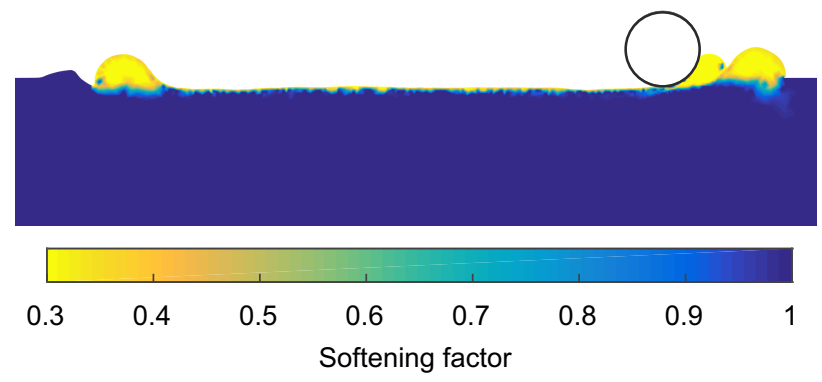

Fig. 5. Softening factor for soil strength in Test A: sweep 3, $u / D=7.5$ 


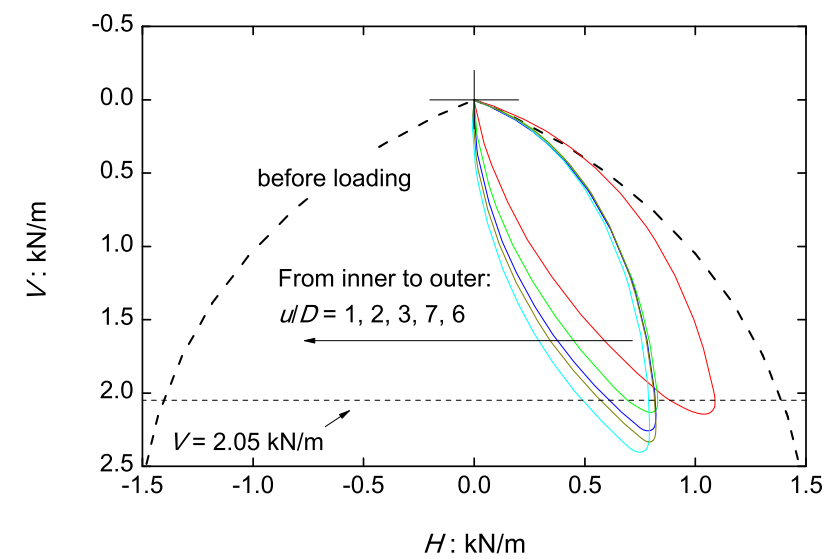

(a)

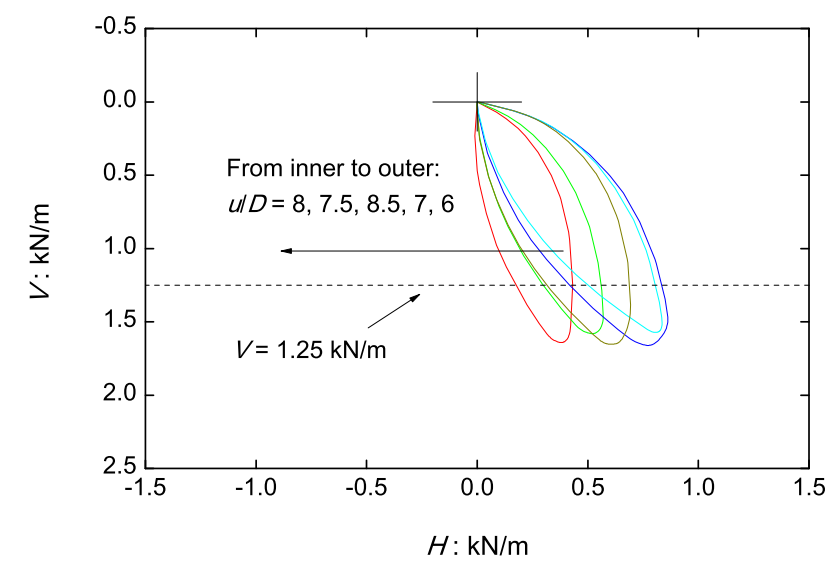

(b)

Fig. 6. Evolution of $V-H$ failure envelope in test A: (a) sweep 1; (b) sweep 3 


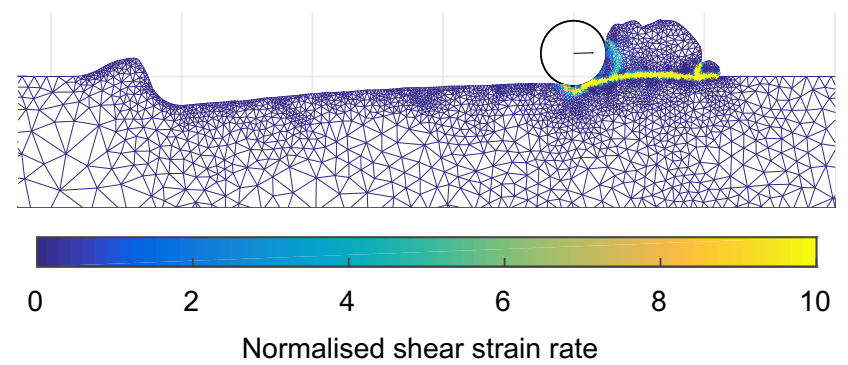

(a)

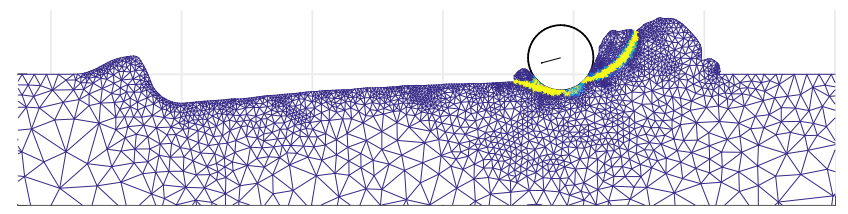

(b)

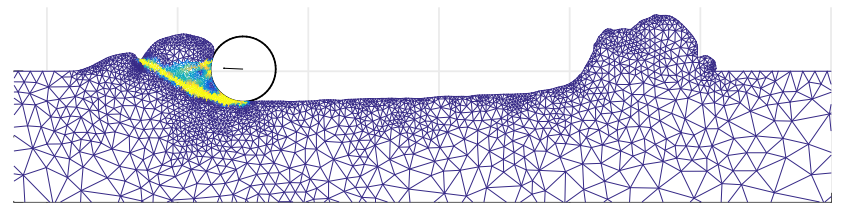

(c)

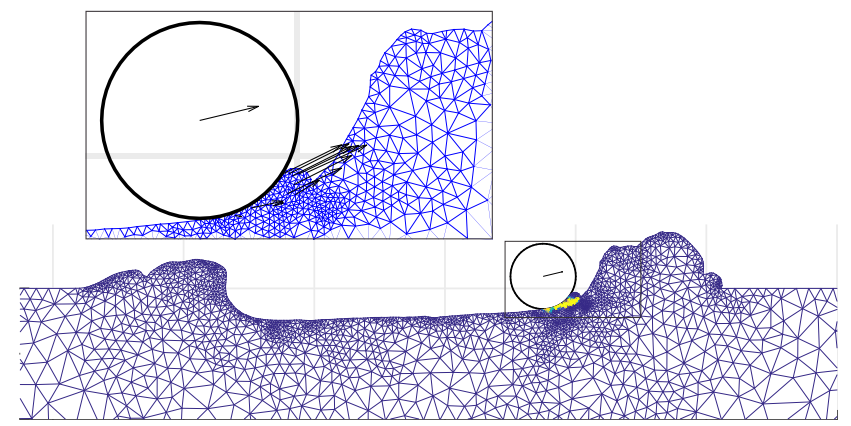

(d)

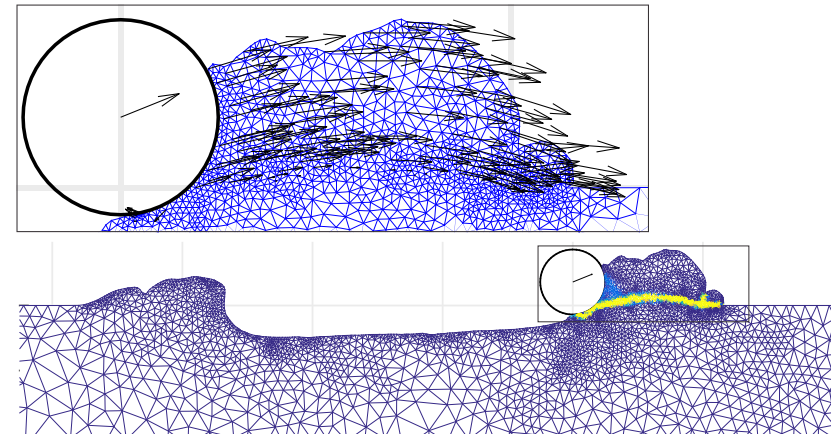

(e)

Fig. 7. Normalised shear strain rate $\left(\dot{\gamma} D / v_{p}\right)$ and velocity field in Test B: (a)-(e) correspond to points B1-B5 in Fig. 3(d)

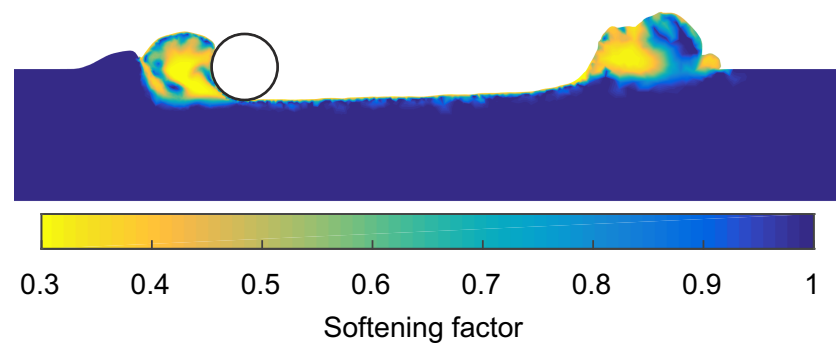

Fig. 8. Softening factor for soil strength in Test B: sweep 2, $u / D=1.0$ 


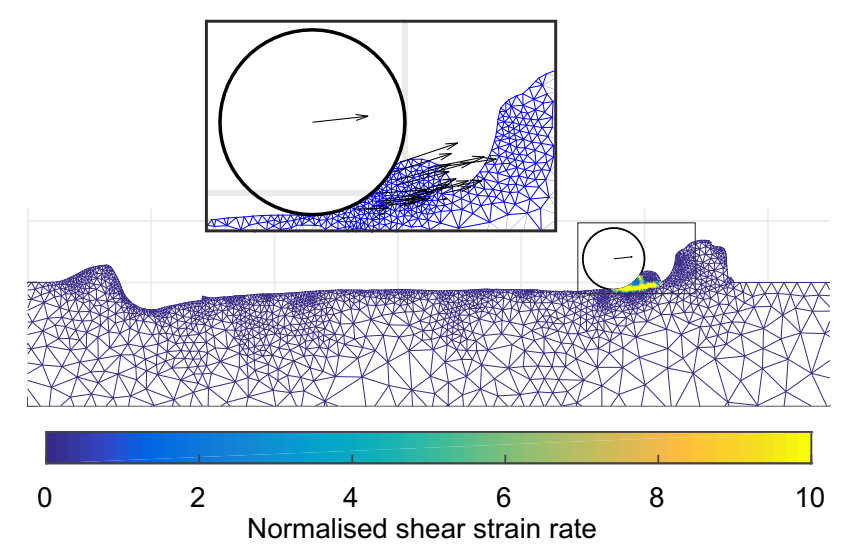

(a)

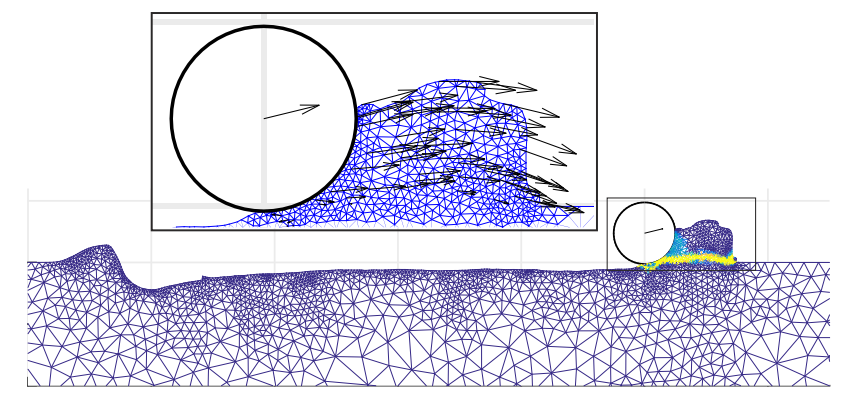

(b)

Fig. 9. Normalised shear strain rate $\left(\dot{\gamma} D / v_{p}\right)$ and velocity field in Test C: (a) and (b) correspond to points $\mathbf{C 1}$ and $\mathbf{C 2}$ in Fig. 3(f)

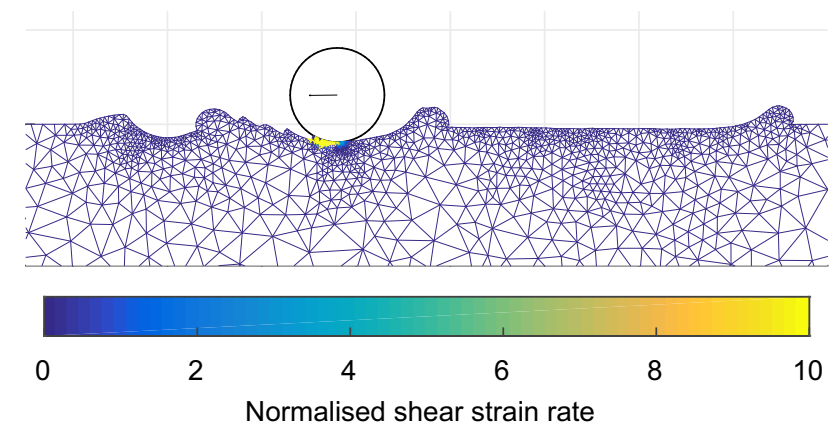

(a)

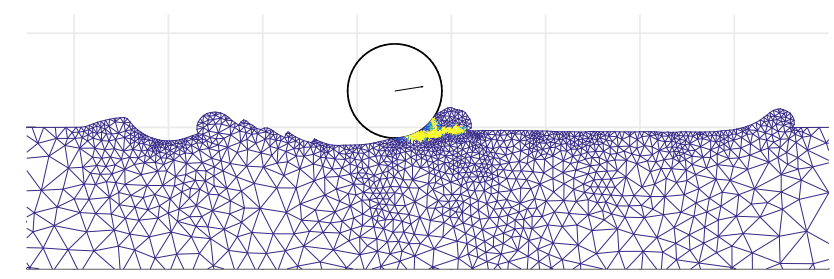

(b)

Fig. 10. Normalised shear strain rate $\left(\dot{\gamma} D / v_{p}\right)$ in Test D: (a) and (b) correspond to points D1 and D2 in Fig. 3(g) 


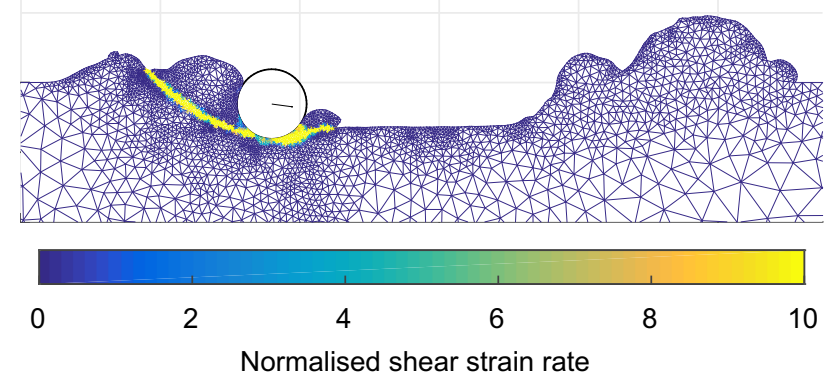

(a)

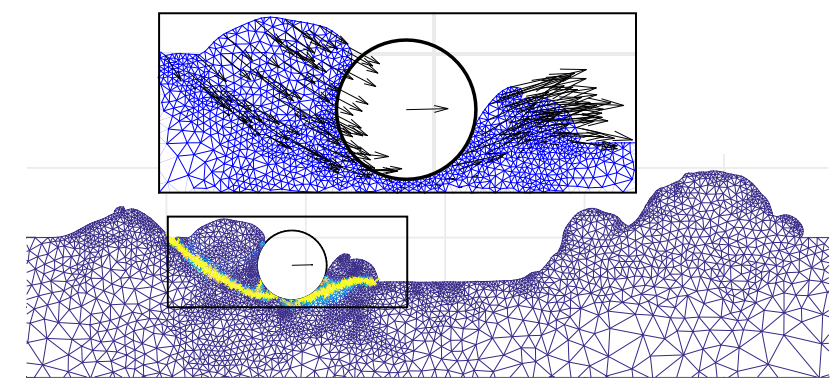

(b)

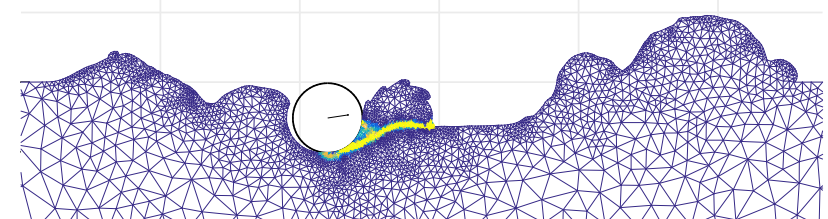

(c)

Fig. 11. Normalised shear strain rate $\left(\dot{\gamma} D / v_{p}\right)$ and velocity field in Test $\mathbf{E}$ : (a)-(c) correspond to the right extremities during sweeps 3,5 and 11, points E1-E3 in Fig. 3(i)

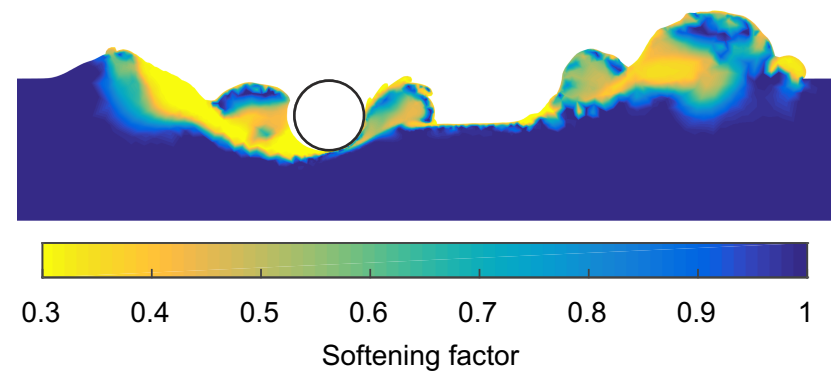

Fig. 12. Softening factor for soil strength in Test E: sweep 11, right extremity 


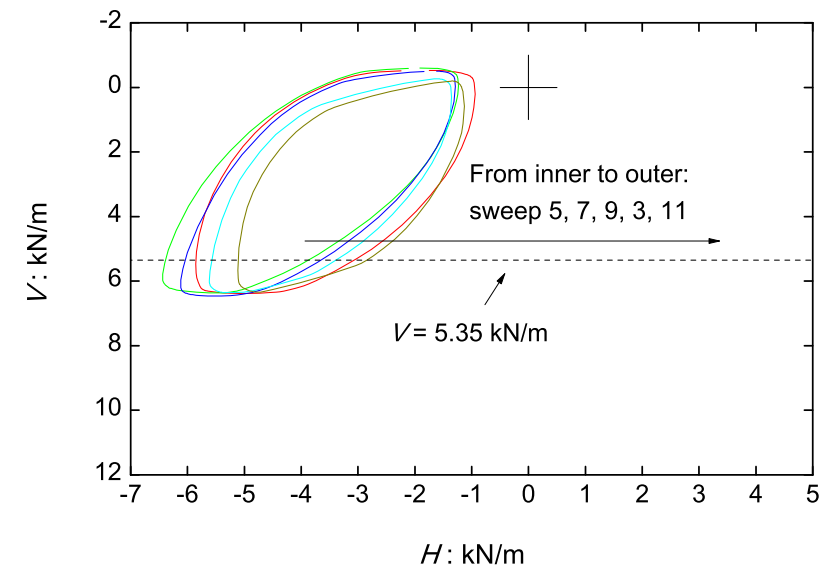

(a)

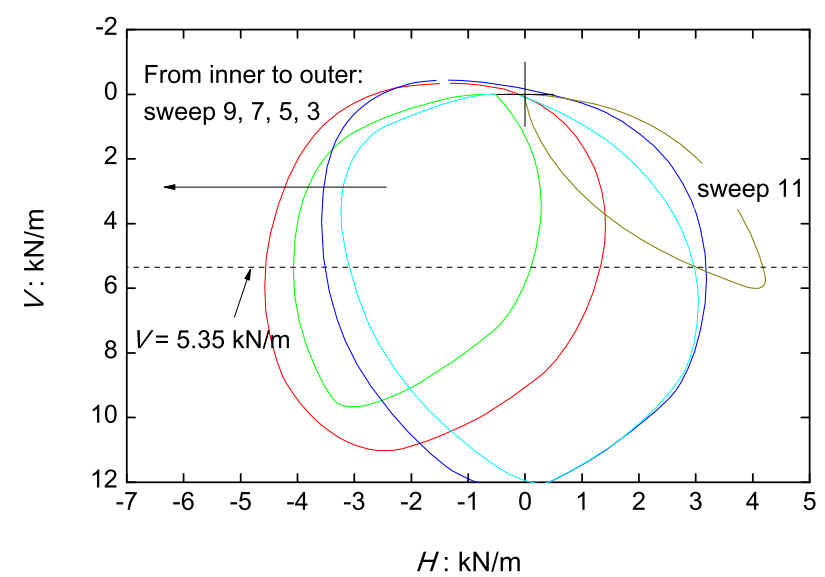

(b)

Fig. 13. Evolution of $V-H$ failure envelope in Test $\mathbf{E}$ during sweeps 3-11: (a) left extremities; (b) right extremities 


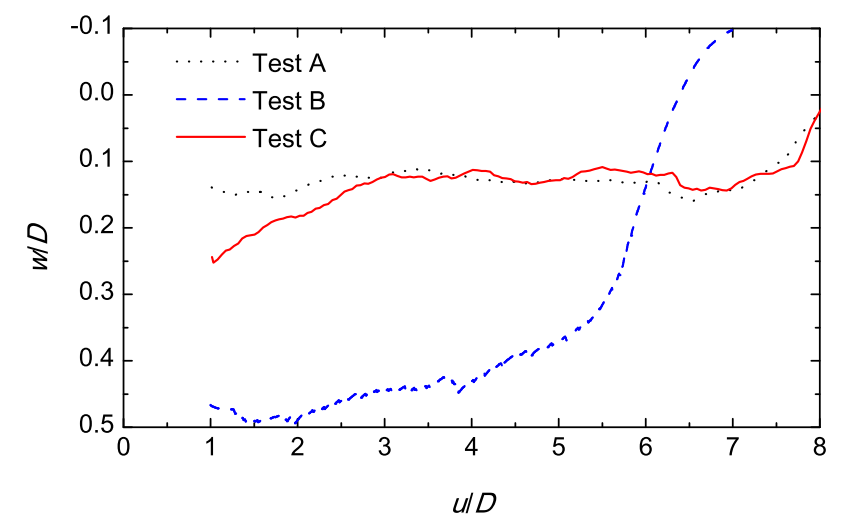

(a)

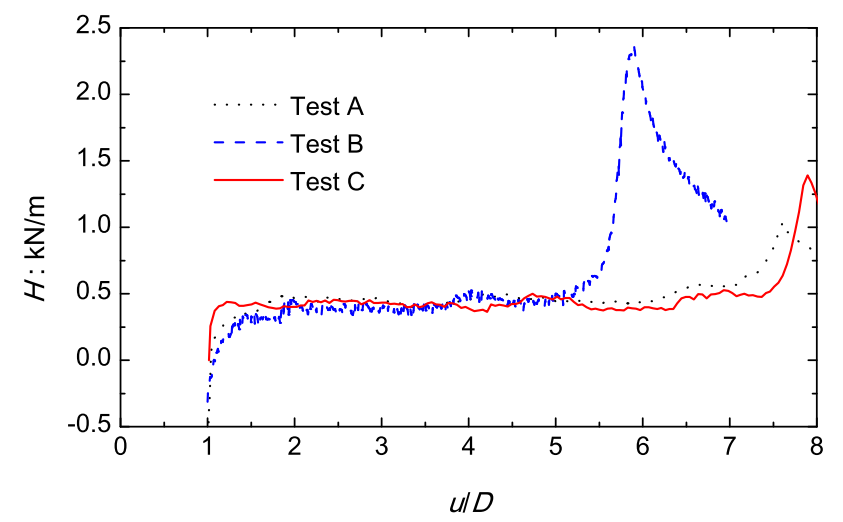

(b)

Fig. 14. Comparisons of SLA simulations of Tests A, B and C during sweep 3: (a) pipe invert trajectory; (b) lateral resistance 


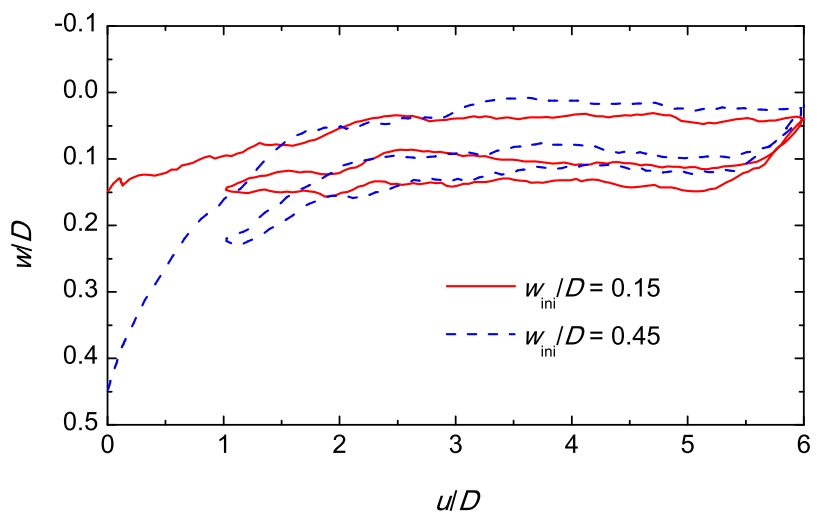

(a)

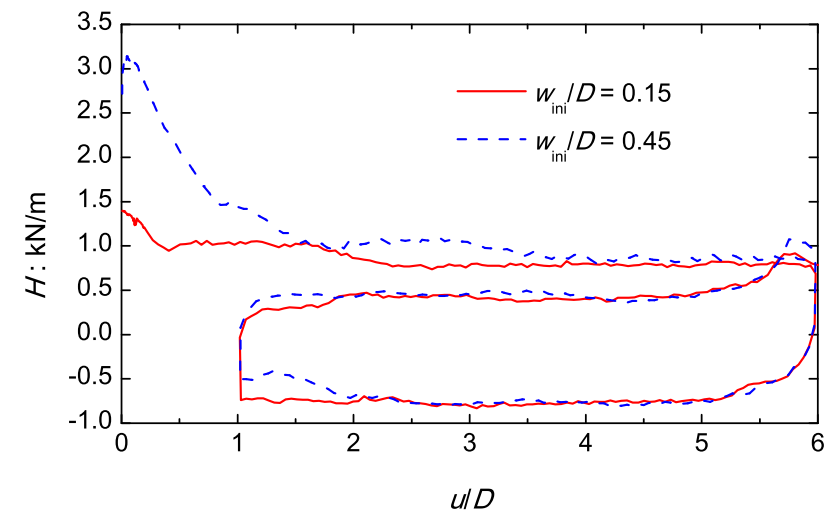

(b)

Fig. 15. Effect of initial embedment on cyclic lateral loading response: (a) pipe invert trajectory; (b) lateral resistance. For all analyses $V_{1}=V_{2}=2.05 \mathbf{k N} / \mathbf{m}, V_{3}=1.25 \mathbf{~ k N} / \mathbf{m}$ 


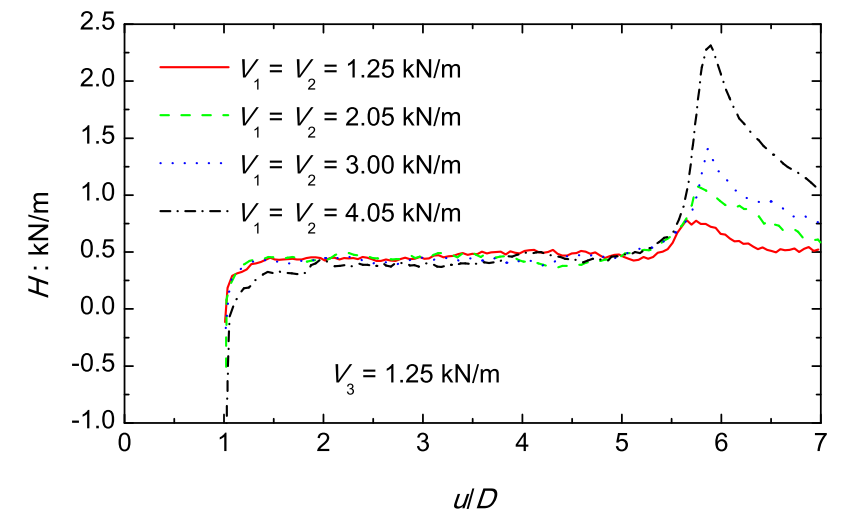

(a)

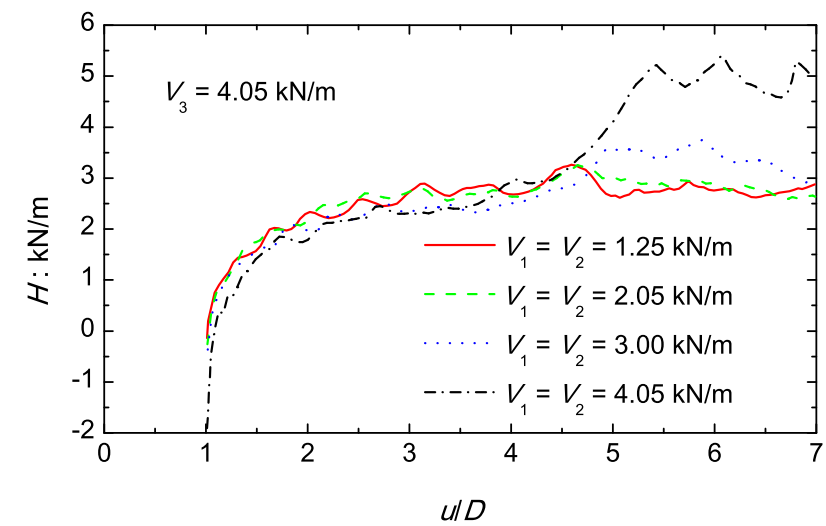

(b)

Fig. 16. Effect of previous vertical loading history on lateral resistance during sweep 3: (a) over-penetrated pipe; (b) normally-penetrated pipe. For all analyses $w_{\text {ini }} / D=0.45$ 